\title{
Potentiation of noise-induced hearing loss by amikacin in guinea pigs
}

\author{
Ching-Ting Tan a,b, Chuan-Jen Hsu b, Shiann-Yann Lee b, Shing-Hwa Liu a , \\ Shoei-Yn Lin-Shiau a,c,* \\ a Institute of Toxicology, College of Medicine, National Taiwan University, No. 1, Jenai Road, Section 1, Taipei 10043, Taiwan \\ b Department of Otolaryngology, National Taiwan University Hospital, Taipei, Taiwan \\ c Institute of Pharmacology, College of Medicine, National Taiwan University, Taipei, Taiwan
}

Received 5 April 2001; accepted 19 July 2001

\begin{abstract}
Noise and aminoglycosides initially attack cochlear outer hair cells (OHCs). Distortion product otoacoustic emissions (DPOAEs) are used for the early diagnosis of damage to OHCs. The effects of sub-damaging doses of amikacin, an aminoglycoside antibiotic agent, on noise-induced hearing loss (NIHL) were examined in guinea pigs. Animals were grouped by gender and exposed to broadband noise at $105 \mathrm{~dB}$ SPL for $12 \mathrm{~h}$ and/or injected i.m. with either amikacin ( $100 \mathrm{mg} / \mathrm{kg} / \mathrm{day})$ or saline for 10 days. Auditory brainstem response (ABR) thresholds, along with DPOAE amplitudes, were measured serially before and after noise exposure. DPOAE amplitudes decreased and ABR thresholds elevated immediately after noise exposure and then gradually recovered. At all frequencies, the emission amplitudes recovered completely to pre-exposure baseline values by 4 days after noise exposure. There was no effect of amikacin on either the ABR threshold or DPOAE amplitudes, in animals treated with amikacin only. However, amikacin significantly prolonged the effect of noise exposure on DPOAE amplitude but not on the noise-induced temporary threshold shift (TTS) of the ABR. In animals treated with a combination of noise and amikacin, significant changes in DPOAE amplitudes were still observed at 4 weeks after cessation of noise exposure. No gender difference in the responses to noise and/or amikacin could be demonstrated. The present findings indicate that even sub-damaging dosages of amikacin might impair recovery from NIHL in guinea pigs. (C) 2001 Elsevier Science B.V. All rights reserved.
\end{abstract}

Key words: Distortion product otoacoustic emission; Noise; Amikacin; Outer hair cell; Guinea pig

\section{Introduction}

Cochlear outer hair cells (OHCs) are known to be the

\footnotetext{
* Corresponding author. Tel.: +886 (2) 23123456/8606; Fax: +886 (2) 23410217

E-mail address: syl@ha.mc.ntu.edu.tw (S.-Y. Lin-Shiau).
}

Abbreviations: OHCs, outer hair cells; DPOAE, distortion product otoacoustic emission; ABR, auditory brainstem response; TTS, temporary threshold shift; NIHL, noise-induced hearing loss; OAE, otoacoustic emission; $f_{1}$, the lower pure primary tone used for DPOAE measurements; $f_{2}$, the higher pure primary tone used for DPOAE measurements; DP-gram, a graph of DPOAE amplitude (in $\mathrm{dB}$ SPL) as a function of stimulus frequency ( $f_{2}$ in this study); SPL, sound pressure level; DSP, digital signal processing; MN1, male guinea pigs treated with noise plus normal saline; FN1, female guinea pigs treated with noise plus normal saline; MA10, male guinea pigs treated with amikacin; FA10, female guinea pigs treated with amikacin; MN1A10, male guinea pigs treated with noise plus amikacin; FN1A10, female guinea pigs treated with noise plus amikacin; GDNF, glial cell line-derived neurotrophic factor \section{.}


measurements) $-f_{2}$ (the higher pure primary tone used for DPOAE measurements) DPOAE testing is preferable to click-evoked auditory brainstem response (ABR) testing for early detection of gentamicin toxicity of the cochlea (Shi and Martin, 1997). In this study, a wider frequency range of DPOAEs was used for investigating OHCs of different turns of the cochlea.

Synergistic toxic effects of noise and aminoglycoside antibiotics on auditory function have been reported by other investigators (Jauhiainen et al., 1972; Brown et al., 1978, 1985; Brummett et al., 1992), with the dosage of aminoglycoside antibiotic administered alone being large enough to produce a small amount of measurable permanent damage, and the sound alone also intense enough to do so. It is difficult to determine if an interaction might occur and result in small or even no permanent ototoxic effects when lower doses of an ototoxic drug and moderate acoustic trauma are administered. In addition, under clinical conditions, patients who need to receive aminoglycoside antibiotics for treatment of their infections usually are not exposed to loud noises during the therapeutic period. It is thus important to know whether low doses of aminoglycoside antibiotics are harmful to patients who have been previously exposed to noise. Therefore, in this study, we attempted to examine the effect of typical clinical uses of amikacin on moderate, reversible, noise-induced hearing loss (NIHL) in guinea pigs.

A previous study in rats showed that there is a gender difference in the susceptibility to aminoglycoside-induced ototoxicity (Mills et al., 1999). Treatment with kanamycin $(400 \mathrm{mg} / \mathrm{kg} /$ day) for 13 consecutive days caused changes in all test frequencies of DPOAEs in male rats. However, significant changes were found in female rats only after treatment with the same daily dose of kanamycin for 22 consecutive days. Considering that the usual clinical uses of aminoglycoside antibiotics are at lower dosages and that the duration of therapy lasts for 10-14 days, we investigated whether a lower dose $(100 \mathrm{mg} / \mathrm{kg} /$ day) and a shorter duration (10 days) of aminoglycosides would have a measurable effect on DPOAEs and whether there is a gender difference. In addition, the gender effect on the ototraumatic interaction between amikacin and acoustic exposure was also studied.

The objectives of this study were to investigate and compare: (1) the effect of noise or a lower dose of amikacin on DPOAEs; (2) whether amikacin modifies the temporary effects of noise on cochlear function measured as TTS of ABR and changes in DPOAE amplitude; and (3) whether there is a gender difference in the ototoxic effects caused by amikacin alone or in combination with noise.

The main findings of this study indicate that amika- cin at sub-damaging doses impaired the recovery of NIHL, thus converting reversible into irreversible changes of DPOAE amplitude. This suggests that physicians need to be cautious when using aminoglycoside antibiotics to treat patients who have previously been exposed to noise.

\section{Materials and methods}

The use and care of animals reported in this study were approved by National Taiwan University's Animal Care and Use Committee.

\subsection{Animals}

Thirty-six guinea pigs weighing $250-300$ g with Preyer's reflexes and normal ABR thresholds $(<25 \mathrm{~dB}$ sound pressure level (SPL)) were used in this experiment. The animals were free from both illness and middle ear infections at the time of the study.

Animals were grouped by gender and treatment as follows: (1) male guinea pigs treated with noise plus normal saline (MN1), (2) female guinea pigs treated with noise plus normal saline (FN1), (3) male guinea pigs treated with amikacin (MA10), (4) female guinea pigs treated with amikacin (FA10), (5) male guinea pigs treated with noise plus amikacin (MN1A10), and (6) female guinea pigs treated with noise plus amikacin (FN1A10), with $n=6$ for each group.

\subsection{DPOAE measurements}

Measurements were performed in a double-walled sound booth (Shing Ho Acoustic Technical Engineering, Taipei, Taiwan). DPOAEs were elicited by two continuous, pure primary tones at frequencies $f_{1}$ and $f_{2}\left(f_{2}: f_{1}\right.$ was fixed at 1.22$)$, generated by two separate transducers (ER2; Etymotic Research, Elk Grove Village, IL, USA) connected to a digital signal processing (DSP) board (SmartOAE DSP Board for Windows; Intelligent Hearing Systems, Miami, FL, USA). Two short plastic tubes connected the transducer outputs to the OAE probe (ER10B+; Etymotic Research), which also contained a miniature low-noise microphone for emission detection. The tapered end of the probe was extended with a short, soft silicon tip $(2 \mathrm{~mm}$ long); it was deeply and tightly inserted into the external ear canal. The microphone of the probe system detected the overall acoustic signal in the external ear canal during tone stimulation. Its response was preamplified $(+40 \mathrm{~dB})$ then analyzed by the SmartOAE DSP board. The entire stimulation and detection process was automatically controlled using a PC computer driven 
by SmartOAE software (Intelligent Hearing Systems, version 3.72). This software, based on Fourier transformation calculation, generated the stimulations through two independent channels, checked the actual levels of stimulating tones, and computed the complex response at frequency $2 f_{1}-f_{2}$. The spectral resolution of each frequency data point was $4.88 \mathrm{~Hz}$. The stimulus parameters were chosen according to published data about DPOAEs (Probst et al., 1991). The testing frequency range of $f_{2}$ was $1.1-17.7 \mathrm{kHz}$ with four frequencies per octave. The two primaries, $L_{1}$ and $L_{2}$, were set 65 and $55 \mathrm{~dB}$ SPL. Amplitudes of DPOAEs were measured at selected frequencies. A graph of DPOAE amplitude (in dB SPL) as a function of stimulus frequency ( $f_{2}$ in this study) (DP-gram), was used for reporting DPOAE findings. Data are described with respect to $f_{2}$ frequency since the generator site of the $2 f_{1}-f_{2}$ distortion product has been most closely correlated with the $f_{2}$ frequency place in the cochlea (Brown and Kemp, 1984).

\subsection{ABR measurements}

The hearing status of all animals was evaluated with click-evoked ABR as described previously (Hsu et al., 2000). In brief, an evoked potential system (Nicolet, Spirit, Madison, WI, USA) was used to measure the thresholds of the ABR. The response signals were recorded by subcutaneous needle electrodes. The active electrodes were placed in the vertex and the ipsilateral retro-auricular region with a ground electrode on the neck of the animal. Click sounds (57.7/s) were used to evoke an ABR. The intensity of the stimulus was varied in 5-dB stepwise increments. Threshold was defined as the lowest intensity level at which a response was still visible. At threshold intensity, at least two sequences of recordings were made to confirm response reproducibility.

\subsection{Noise exposure}

Noise-exposed animals were exposed to broadband noise at around $105 \mathrm{~dB}$ SPL for $12 \mathrm{~h}$. During noise exposure, animals were kept in a cage inside a soundproof room. The broadband noise was produced by an audiometer (Grason-Stadler, model GSI-10, Milford, NH, USA) and presented by a set of loudspeakers on both sides of the cage. The frequency ranges were from $32 \mathrm{~Hz}$ to $20 \mathrm{kHz}$. The SPL of the noise was measured with a sound level meter (Nion, model NA-24, Tokyo, Japan) situated on the cage at the onset and upon completion of noise exposure. The sound intensity varied by $3 \mathrm{~dB}$ throughout the exposure field and was measured as $105 \mathrm{~dB}$ at the center of the chamber where subjects were placed.

\subsection{Experimental procedures}

Noise exposure was presented during the first $12 \mathrm{~h}$ of day 0. Amikacin (amikacin sulfate, Banyu, Tokyo, Japan), $100 \mathrm{mg} / \mathrm{kg} / \mathrm{day}$, or an equivalent volume of saline was administered i.m. to animals starting from day 0 for 10 consecutive days. The control groups were not exposed to noise.

ABR thresholds in response to clicks as well as amplitudes of DPOAEs were obtained in anesthetized animals before noise exposure and/or amikacin administration and on days $0,1,2,4,7,9,14$, and 21 , as well as on the final 28th day.

\subsection{Statistical analysis}

Mean DPOAE measurements were calculated for each group and separated by frequency levels. Comparisons among the same test group were made by one-way repeated measures analysis of variance followed by
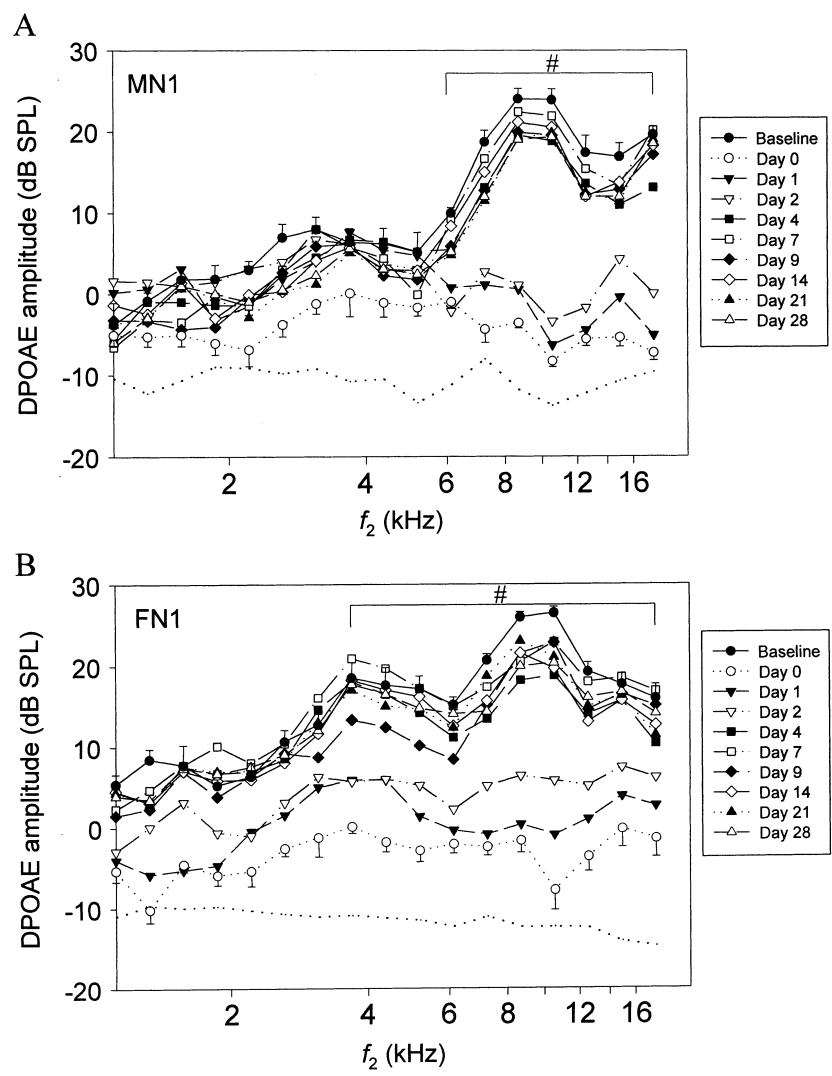

Fig. 1. Changes in amplitudes of DPOAEs after noise exposure (105 dB SPL, $12 \mathrm{~h}$ ) in male (A) and female (B) guinea pigs. Stimulus levels were $L_{1}=65$ and $L_{2}=55 \mathrm{~dB}$ SPL. Error bars, representing + or -1 S.E.M., are included only for the baseline and day 0 (immediate post-exposure curves in the plots). The dotted line at the bottom of each graph represents the noise floor of the emission recording system. ${ }^{\#} P<0.05$ (indicates a statistically significant difference from the baseline for the first 3 days after noise exposure, as explained in the text). 
Dunnett's test when appropriate. A $P$ value of less than 0.05 was considered statistically significant. For results shown in Figs. 1-3, error bars, representing + or -1 S.E.M., are only shown for the baseline and day 0 (immediate post-exposure curves in the plots). For results shown in Fig. 4, data are presented as mean \pm S.E.M. For results shown in Fig. 5, error bars are not included. These presentations of results are for the purpose of clarity.

\section{Results}

Fig. 1 shows the effect of 12-h noise exposure on DPOAE amplitudes in male (the MN1 group) and female (the FN1 group) animals. As shown, both the MN1 and FN1 groups displayed a maximal depression of DPOAE amplitudes on day 0 immediately after noise exposure which gradually returned to the baseline in 4 days. Statistical analyses across all test frequencies of DPOAEs between the baseline and day 4 recovery data indicate no statistical differences in DPOAE amplitudes for most of the frequencies tested. Depressions of emissions for $f_{2}$ were noted for the first three post-
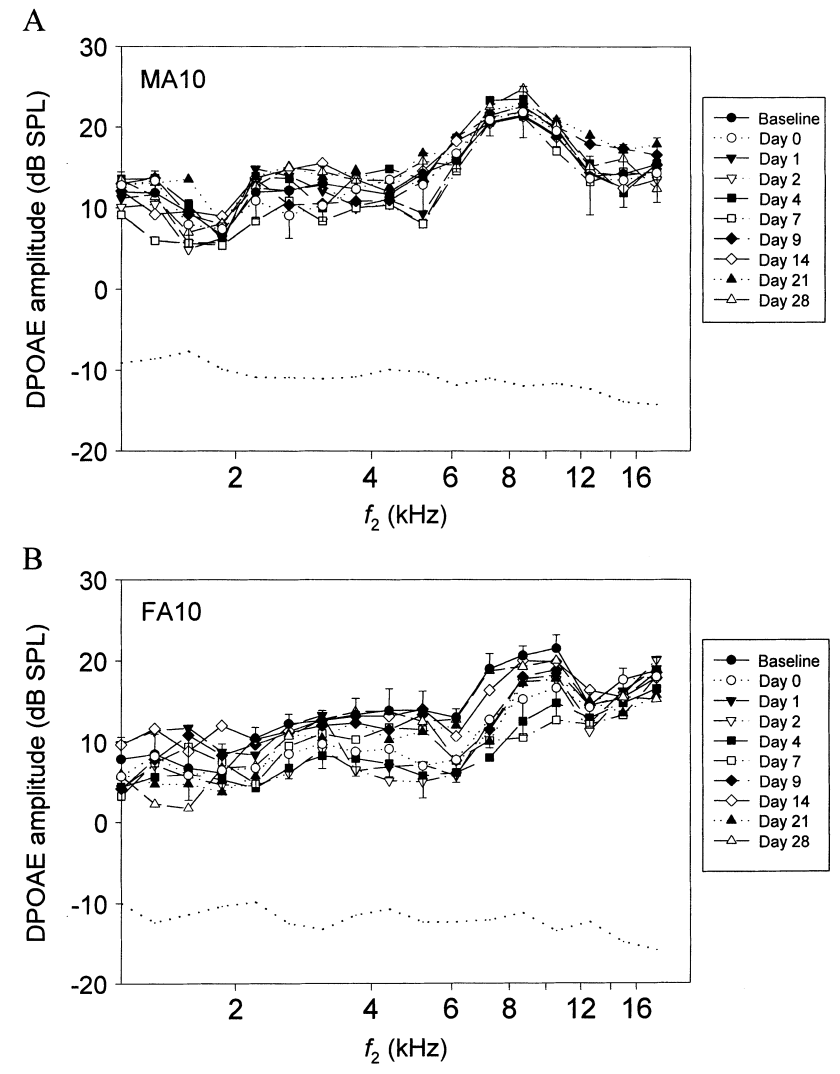

Fig. 2. Changes in amplitudes of DPOAEs induced by amikacin $(100 \mathrm{mg} / \mathrm{kg}$, i.m., for 10 consecutive days) in male (A) and female (B) guinea pigs. Stimulus levels, error bars, and the dotted line are explained in the legend for Fig. 1.
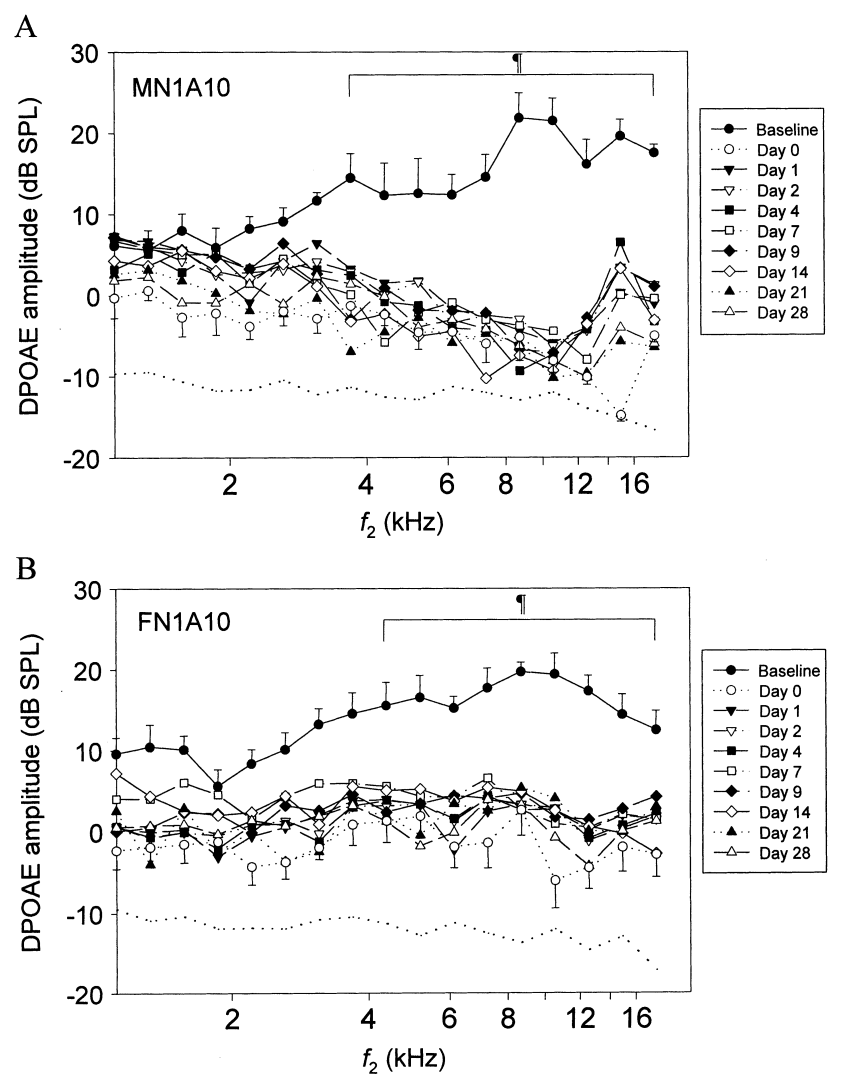

Fig. 3. Changes in amplitudes of DPOAEs induced by a combination of noise and amikacin in male (A) and female (B) guinea pigs. Stimulus levels, error bars, and the dotted line are explained in the legend for Fig. 1. ${ }^{\text {" }} P<0.05$ (indicates a statistically significant difference from the baseline for all tested days after noise exposure, as explained in the text).

exposure days from 6.3 to $17.7 \mathrm{kHz}$ in the $\mathrm{MN} 1$ group and from 3.7 to $17.7 \mathrm{kHz}$ in the $\mathrm{FN} 1$ group.

Fig. 2 shows the effect of amikacin on DPOAE amplitudes in male (the MA10 group) and female (the FA10 group) animals. As shown, amikacin, at the doses used in this study, caused no significant changes in DPOAE amplitudes for $f_{2}$ in the frequency range tested in either the MA10 or FA10 groups.

Fig. 3 shows the effect of a combination of noise exposure and amikacin on DPOAE amplitudes in male (the MN1A10 group) and female (the FN1A10 group) animals. As shown, the same noise exposure also caused immediate and significant depressions in DPOAE amplitudes for $f_{2}$ on day 0 in both the MN1A10 and FN1A10 groups. However, unlike the recovery found in animals receiving noise exposure only, depressions of DPOAE amplitudes persisted until the end of the 28-day observation period in both study groups. Consequently, depressions of emissions for $f_{2}$ were observed from 3.7 to $17.7 \mathrm{kHz}$ in the MN1A10 group and from 4.4 to $17.7 \mathrm{kHz}$ in the FN1A10 group for all tested days. 


\begin{tabular}{|lll|}
\hline MN1 & MA10 & $\rightleftharpoons$ MN1A10 \\
FN1 & FA10 & FाIIII FN10 \\
\hline
\end{tabular}

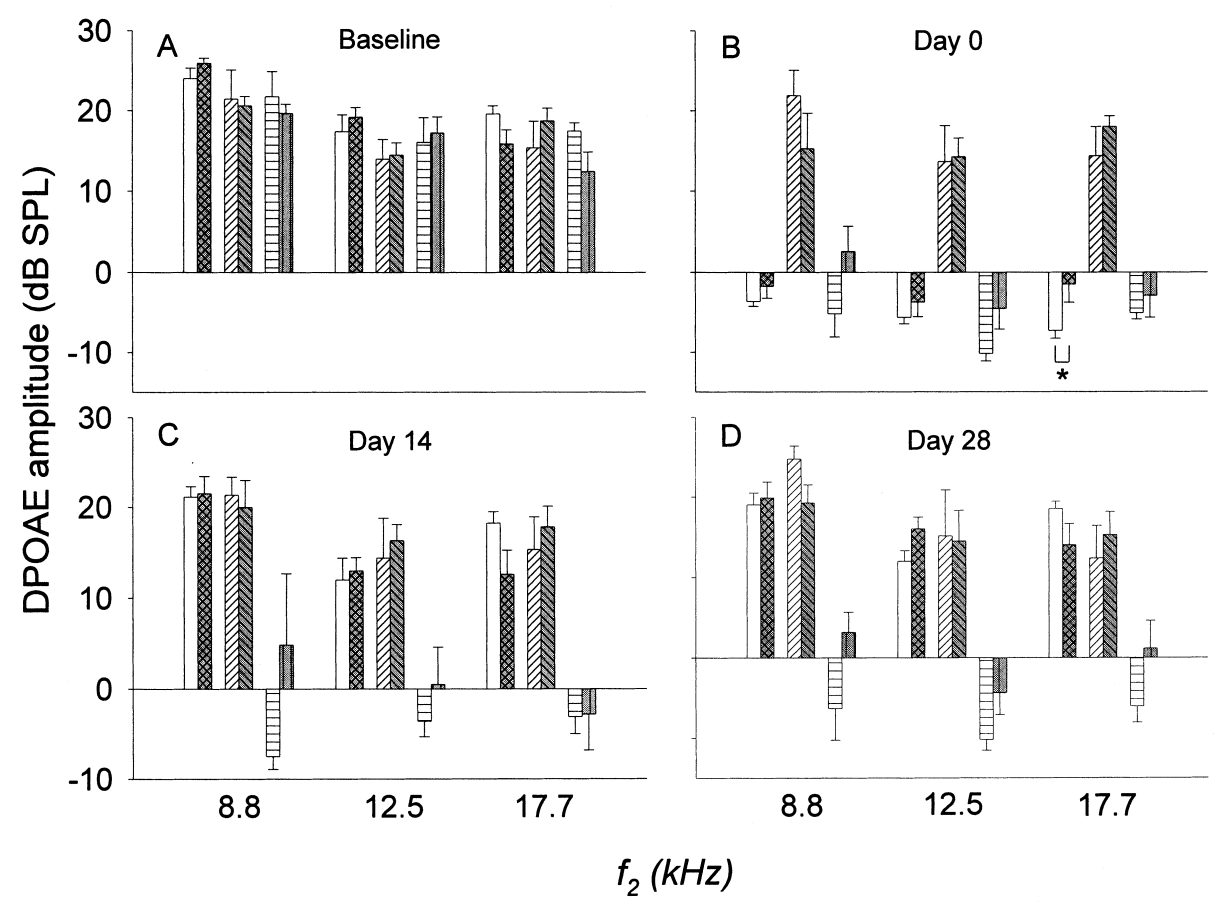

Fig. 4. Changes of DPOAE amplitudes for $f_{2}$ at $8.8,12.5$, and $17.7 \mathrm{kHz}$ at the baseline (A), and on days 0 (B), 14 (C), and 28 (D). ${ }^{*} P<0.05$ (indicates a statistically significant difference between male and female animals).

Fig. 4 compares gender differences in the effect of noise exposure alone, amikacin alone, or their combination on DPOAE amplitudes for $f_{2}$ at $8.8,12.5$, and $17.7 \mathrm{kHz}$ at the baseline, and on days 0,14 , and 28 . These frequencies and testing days were chosen for comparison because they were in the range of prominent effects induced by these three experimental interventions. As shown, differences between male and female animals in DPOAE amplitudes for $f_{2}$ at any selected frequency on any given day (except $17.7 \mathrm{kHz}$ of noise alone group on day 0) did not reach levels of statistical significance.

Unlike the effects on DPOAE amplitudes, these experimental interventions had different influences on the threshold shift of ABR, another test commonly used for auditory functions. Fig. 5 shows the threshold shift of $A B R$ in various study groups over time. As shown, the threshold shift of ABR was observed in all noise exposure groups (the MN1, FN1, MA10N1, and FA10N1 groups), but not in non-exposure groups (MA10, FA10). The ABR thresholds significantly increased after noise exposure on day 0 and gradually recovered to their baselines within 4 days. Although the ABR thresholds on days 1 and 2 in the noise exposure groups seemed to be higher than their baselines, no statistical significance could be detected. Amikacin alone caused no significant changes in ABR thresholds for the entire 28-day observation period after the first dose in the MA10 and FA10 groups.

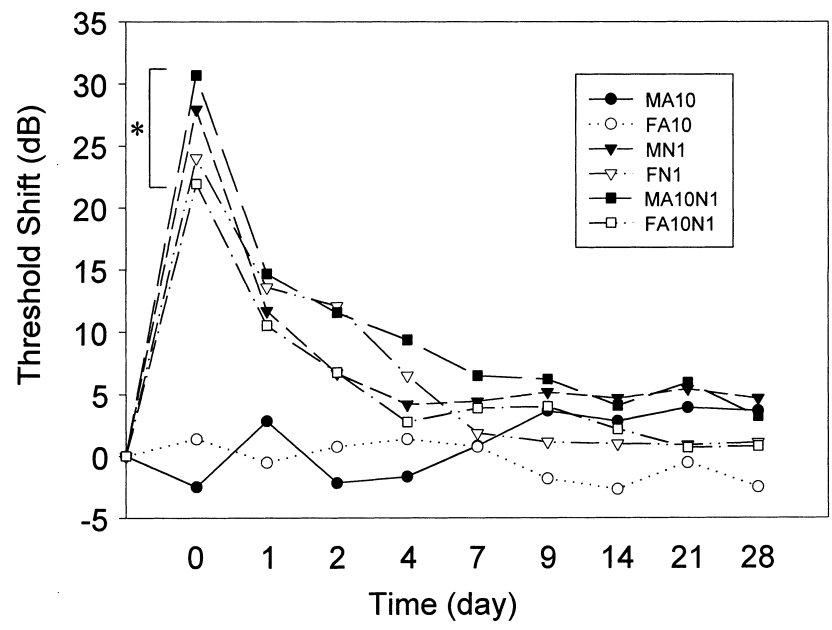

Fig. 5. ABR threshold shifts in guinea pigs after noise exposure and/or amikacin administration. For noise and/or amikacin groups (MN1, FN1, MA10N1, FA10N1), ABR thresholds significantly increased on the day of noise exposure. ${ }^{*} P<0.05$ (indicates a statistically significant difference from the baseline). 


\section{Discussion}

Results of this study clearly demonstrate that DPOAEs are more sensitive than click-ABR in detecting NIHL at higher frequencies. An ideal test for identifying shifts in cochlear function should be highly repeatable and sensitive to minor damage. A previous study concluded that various OAE measures have the potential to distinguish small changes in cochlear function, and hence show promise for monitoring cochlear function in ears exposed to noise or other hazards (Hall and Lutman, 1999). The objectivity of the measurements and the short test time required further enhance the usefulness of OAEs as a cochlear measure (Attias and Bresloff, 1996). Continuous noise exposure has been shown to cause a reduction in DPOAE amplitudes (Eddins et al., 1999). However, in a number of cases, emission shifts were unaccompanied by notable TTSs of ABR. Therefore, it appears that emissions are more sensitive to $\mathrm{OHC}$ changes and more appropriate for screening and monitoring ears at risk of NIHL.

DPOAE is also a sensitive measure of drug-induced hearing loss (Brown et al., 1985). Ototoxic amikacin treatment in a chinchilla model $(400 \mathrm{mg} / \mathrm{kg} /$ day, i.m.) revealed that high-frequency components of DPOAEs change first, followed by lower-frequency components (Kakigi et al., 1998). In this study, although wide frequency ranges of DPOAEs were checked under 65/55 dB SPL stimuli intensities, no significant changes in amplitude of DPOAEs could be demonstrated in amikacin-treated animals, suggesting that the dose of amikacin used was a sub-damaging dose. Likewise, since the changes found in both the MN1A10 and FN1A10 groups were at the higher frequency ranges, these results lend additional evidence to suggest that measurements of DPOAE amplitudes are more sensitive in detecting ototoxic effects of OHCs than are traditional audiometric examinations.

In the present study, noise exposure alone resulted in only temporary changes of DPOAE amplitudes. However, a combination of noise exposure and a sub-damaging dose of amikacin caused marked decreases in DPOAE amplitudes from about the $4-17.7-\mathrm{kHz}$ range, which persisted for as long as 28 days after the acoustic insult. These results suggest that application of amikacin may impair the recovery of hearing loss from noise exposure. This aggravated situation is not unique because carbon monoxide (Fechter et al., 1987; Young et al., 1987; Fechter et al., 1988) and toluene (Johnson et al., 1988, 1990; Morata et al., 1993; Lataye and Campo, 1997) have also been shown to aggravate NIHL. The mechanisms underlying this aggravation of noiseinduced $\mathrm{OHC}$ damage by aminoglycosides are not yet known. Several possibilities can be suggested. First of all, the involvement of medial olivocochlear efferents should be considered. It has been shown that protection of the ipsilateral ear from acoustic trauma occurs when the contralateral ear is simultaneously stimulated at the same frequency, but at a lower intensity (Cody and Johnstone, 1982). This protective effect can be blocked by strychnine, a known blocker of the medial efferent system of the inner ear (Puel et al., 1988). Since even a single injection of aminoglycosides can result in rapid elimination of function of this pathway (Smith et al., 1994), whether the impaired functioning of medial olivocochlear efferents during the application of aminoglycoside interferes with the recovery of $\mathrm{OHC}$ function after acoustic trauma needs to be clarified.

Another possibility for the impairment of recovery from noise exposure by aminoglycoside is that both of these two factors can increase free radical levels (Yamane et al., 1995; Clerici et al., 1996; Hirose et al., 1997; Lopez-Gonzalez et al., 1999; Sha and Schacht, 1999), which may result in auditory damage (Liu, 1992). Recently, protection against NIHL or aminoglycoside ototoxicity has been shown by inhibition of free oxygen radical-induced lipid peroxidation (Quirk et al., 1994) or by free radical scavengers (Hester et al., 1998; Yamasoba et al., 1999; Ohinataab et al., 2000; Sha and Schacht, 2000). Since the potential cytotoxic effects of free radicals are mitigated by several antioxidant enzymes and scavengers in living tissues, the overall levels of free radicals in living tissues are determined by the balance between the production of free radicals and the cellular antioxidant defense capacity. Amikacin administered immediately after noise trauma might further disturb this balance and impair the recovery from NIHL.

The third possibility is that both noise exposure and aminoglycosides might disturb calcium homeostasis. In guinea pigs, acute acoustic trauma caused morphological changes of cells in the organ of Corti and are correlated with changes in cellular calcium distribution (Maurer et al., 1993). The authors suggested the possible role of calcium in cell damage and necrosis after acute noise trauma. In addition, previous studies (Corrado et al., 1989; Schacht, 1993; Tan et al., 2001) revealed that calcium is involved in a possible mechanism of the acute ototoxic effects of aminoglycosides on OHCs. The role of disturbed intracellular calcium homeostasis by the combination of noise and amikacin is worthy of clarification.

The fourth possibility is that both noise and aminoglycoside-induced ototoxicity might be, in part, an excitotoxic process involving activation of $N$-methyl-D-aspartate (NMDA) receptors (Segal and Skolnick, 1998; Harvey and Skolnick, 1999; Masuko et al., 1999; Segal et al., 1999; Jager et al., 2000). NMDA antagonists have been shown to be involved in protection against noise- or aminoglycoside-induced ototoxicity (Basile et 
al., 1996; Duan et al., 2000). It is thus plausible that impairment of NIHL by aminoglycosides might involve the augmentation of noise-induced excitotoxicity.

A final possibility is that neurotrophins are not only essential to the survival of neuron cells (Ernfors et al., 1996; Staecker et al., 1996; Miller et al., 1997), but also important in regulating $\mathrm{OHC}$ function. Recently, neurotrophic factors, such as neurotrophin-3 (NT-3), brain-derived neurotrophic factor (BDNF), and glial cell line-derived neurotrophic factor (GDNF) showed differential protective effects in the attenuation of noise-induced (Keithley et al., 1998; Agerman et al., 1999; Yamasoba et al., 1999; Shoji et al., 2000b) or aminoglycoside-induced (Ruan et al., 1999; Yagi et al., 1999) hair cell damage. Since it has been shown that different concentrations of NT-3 or GDNF result in different OHC survival (Shoji et al., 2000a,b), it is possible that in animals exposed to noise and aminoglycosides, changes in the amount of these neurotrophic factors may affect $\mathrm{OHC}$ functions. However, the exact mechanisms of noise-drug interactions remain to be determined.

Although gender susceptibility of kanamycin ototoxicity has been claimed by other authors (Mills et al., 1999), no gender differences were demonstrated in the present study. Amikacin and kanamycin are known to possess similar potency of cochleotoxicity (Basile et al., 1996). Therefore, the discrepancy could be due to the fact that we used different animals (guinea pig vs. rat), lower doses (100 vs. $400 \mathrm{mg} / \mathrm{kg} /$ day) and shorter duration (10 days vs. 13-21 days) of aminoglycoside antibiotic. These experimental findings certainly infer that, for patients, whether male or female, who are likely to be exposed to loud noises in their environments, subsequent aminoglycoside administration could produce ototoxicity.

In summary, amikacin $(100 \mathrm{mg} / \mathrm{kg} /$ day, i.m., for 10 days) caused no significant changes in DPOAE amplitudes or ABR thresholds, while broadband noise (105 $\mathrm{dB}$ SPL, $12 \mathrm{~h}$ ) alone only resulted in TTS of ABR and a temporary decrease of DPOAE amplitudes for 4 days. However, amikacin significantly interfered with the recovery from noise-induced changes in DPOAE amplitudes in guinea pigs treated with a combination of noise exposure and amikacin. The present results indicate that the application of aminoglycosides should be carefully monitored by wider frequency ranges of DPOAEs for those patients who have suffered from moderate noise trauma.

\section{Acknowledgements}

We thank Mr. D.P. Chamberlin for editorial assistance. This work was supported by the National Sci- ence Council of the Republic of China Grants NSC-892314-B-002-238 and NSC-89-2314-B-002-579, and by the National Taiwan University Hospital Grant NTUH-88S1020.

\section{References}

Agerman, K., Canlon, B., Duan, M.L., Ernfors, P., Fredelius, L., 1999. New strategies for preventing hearing loss. In: Prasher, D.K., Canlon, B. (Eds.), Cochlear Pharmacology and Noise Trauma. Noise Research Network Publications, London, pp. 75-84.

Attias, J., Bresloff, I., 1996. Noise induced temporary otoacoustic emission shifts. J. Basic Clin. Physiol. Pharmacol. 7, 221-233.

Basile, A.S., Huang, J.M., Xie, C., Webster, D., Berlin, C., Skolnick, P., 1996. $N$-methyl-D-aspartate antagonists limit aminoglycoside antibiotic-induced hearing loss. Nat. Med. 2, 1338-1343.

Brown, A.M., Kemp, D.T., 1984. Suppressibility of the $2 f_{1}-f_{2}$ stimulated acoustic emissions in gerbil and man. Hear. Res. 13, 2937.

Brown, J.J., Brummett, R.E., Meikle, M.B., Vernon, J.A., 1978. Combined effects of noise and neomycin. Cochlear changes in the guinea pig. Acta Otolaryngol. Stockh. 86, 394-400.

Brown, R.D., Henley, C.M., Penny, J.E., Kupetz, S., 1985. Link between functional and morphological changes in the inner ear: functional changes produced by ototoxic agents and their interactions. Arch. Toxicol. 8 (Suppl.), 240-250.

Brownell, W.E., Bader, C.R., Bertrand, D., de Ribauierre, Y., 1985. Evoked mechanical response of isolated cochlear hair cells. Science 227, 194-196.

Brummett, R.E., Fox, K.E., Kempton, J.B., 1992. Quantitative relationships of the interaction between sound and kanamycin. Arch. Otolaryngol. Head Neck Surg. 118, 498-500.

Canlon, B., Fransson, A., 1995. Morphological and functional preservation of the outer hair cells from noise trauma by sound conditioning. Hear. Res. 84, 112-124.

Clerici, W.J., Hensley, K., DiMartino, D.L., Butterfield, D.A., 1996. Direct detection of ototoxicant-induced reactive oxygen species generation in cochlear explants. Hear. Res. 98, 116-124.

Cody, A.R., Johnstone, B.M., 1982. Temporary threshold shift modified by binaural acoustic stimulation. Hear. Res. 6, 199-205.

Cody, A.R., Russell, I.J., 1985. Outer hair cells in the mammalian cochlea and noise-induced hearing loss. Nature 315, 662-665.

Corrado, A.P., Demorais, I.P., Prado, W.A., 1989. Aminoglycoside antibiotics as a tool for the study of the biological role of calcium ions: historical overview. Acta Physiol. Pharmacol. Lat. Am. 39, 419-430.

Duan, M., Agerman, K., Ernfors, P., Canlon, B., 2000. Complementary roles of neurotrophin 3 and a $N$-methyl-D-aspartate antagonist in the protection of noise and aminoglycoside-induced ototoxicity. Proc. Natl. Acad. Sci. USA 97, 7597-7602.

Eddins, A.C., Zuskov, M., Salvi, R.J., 1999. Changes in distortion product otoacoustic emissions during prolonged noise exposure. Hear. Res. 127, 119-128.

Ernfors, P., Duan, M.L., ElShamy, W.M., Canlon, B., 1996. Protection of auditory neurons from aminoglycoside toxicity by neurotrophin-3. Nat. Med. 2, 463-467.

Fechter, L.D., Thorne, P.R., Nuttall, A.L., 1987. Effect of carbon monoxide on cochlear electrophysiology and blood flow. Hear. Res. 27, 37-45.

Fechter, L.D., Young, J.S., Carlisle, L., 1988. Potentiation of noise induced threshold shifts and hair cell loss by carbon monoxide. Hear. Res. 34, 339-348.

Govaerts, P.J., Claes, J., Van De Heyning, P.H., Jorens, P.G., Mar- 
quet, J., De Broe, M.E., 1990. Aminoglycoside-induced ototoxicity. Toxicol. Lett. 52, 227-251.

Hall, A.J., Lutman, M.E., 1999. Methods for early identification of noise-induced hearing loss. Audiology 38, 277-280.

Harvey, S.C., Skolnick, P., 1999. Polyamine-like actions of aminoglycosides at recombinant $N$-methyl-D-aspartate receptors. J. Pharmacol. Exp. Ther. 291, 285-291.

Hester, T.O., Jones, R.O., Clerici, W.J., 1998. Protection against aminoglycoside otic drop-induced ototoxicity by a spin trap: I. Acute effects. Otolaryngol. Head Neck Surg. 119, 581-587.

Hirose, K., Hockenbery, D.M., Rubel, E.W., 1997. Reactive oxygen species in chick hair cells after gentamicin exposure in vitro. Hear. Res. 104, 1-14.

Hsu, C.J., Shau, W.Y., Chen, Y.S., Liu, T.C., Lin-Shiau, S.Y., 2000. Activities of $\mathrm{Na}^{+}, \mathrm{K}^{+}$-ATPase and $\mathrm{Ca}^{2+}$-ATPase in cochlear lateral wall after acoustic trauma. Hear. Res. 142, 203-211.

Huizing, E.H., de Groot, J.C.M.J., 1987. Human cochlear pathology in aminoglycoside ototoxicity - a review. Acta Otolaryngol. Stockh. 436 (Suppl.), 117-125.

Jager, W., Goiny, M., Herrera-Marschitz, M., Brundin, L., Fransson, A., Canlon, B., 2000. Noise-induced aspartate and glutamate efflux in the guinea pig cochlea and hearing loss. Exp. Brain Res. $134,426-434$.

Jauhiainen, T., Kohonen, A., Jauhiainen, M., 1972. Combined effect of noise and neomycin on the cochlea. Acta Otolaryngol. Stockh. $73,387-390$.

Johnson, A.C., Juntunen, L., Nylen, P., Borg, E., Hoglund, G., 1988. Effect of interaction between noise and toluene on auditory function in the rat. Acta Otolaryngol. Stockh. 105, 56-63.

Johnson, A.C., Nylen, P., Borg, E., Hoglund, G., 1990. Sequence of exposure to noise and toluene can determine loss of auditory sensitivity in the rat. Acta Otolaryngol. Stockh. 109, 34-40.

Kakigi, A., Hirakawa, H., Harel, N., Mount, R.J., Harrison, R.V., 1998. Basal cochlear lesions result in increased amplitude of otoacoustic emissions. Audiol. Neuro-Otol. 3, 361-372.

Keithley, E.M., Ma, C.L., Ryan, A.F., Louis, J.C., Magal, E., 1998. GDNF protects the cochlea against noise damage. Neuroreport 9, 2183-2187.

Kemp, D.T., 1978. Stimulated acoustic emissions from within the human auditory system. J. Acoust. Soc. Am. 64, 1386-1391.

Lataye, R., Campo, P., 1997. Combined effects of a simultaneous exposure to noise and toluene on hearing function. Neurotoxicol. Teratol. 19, 373-382.

Lim, D.J., 1966. Effects of noise and ototoxic drugs at the cellular level in the cochlea: a review. Am. J. Otolaryngol. 7, 73-99.

Liu, Z., 1992. Experimental study on the mechanism of free radical in blast trauma induced hearing loss. Chin. J. Otorhinolaryngol. 27, 24-26.

Lopez-Gonzalez, M.A., Delgado, F., Lucas, M., 1999. Aminoglycosides activate oxygen metabolites production in the cochlea of mature and developing rats. Hear. Res. 136, 165-168.

Masuko, T., Kuno, T., Kashiwagi, K., Kusama, T., Williams, K., Igarashi, K., 1999. Stimulatory and inhibitory properties of aminoglycoside antibiotics at $N$-methyl-D-aspartate receptors. J. Pharmacol. Exp. Ther. 290, 1026-1033.

Maurer, J., Heinrich, U.R., Mann, W., 1993. Morphologic damage and changes of intracellular calcium-binding sites after acute noise trauma in the organ of Corti of the guinea pig. J. Otol-RhinolLaryngol. 55, 7-12.

Miller, J.M., Chi, D.H., O'Keeffe, L.J., Kruszka, P., Raphael, Y., Altschuler, R.A., 1997. Neurotrophins can enhance spiral ganglion cell survival after inner hair cell loss. Int. J. Dev. Neurosci. 15, 631-643.

Mills, C.D., Loos, B.M., Henley, C.M., 1999. Increased susceptibility of male rats to kanamycin-induced cochleotoxicity. Hear. Res. $128,75-79$

Morata, T.C., Dunn, D.E., Kretschmer, L.W., Lemasters, G.K., Keith, R.W., 1993. Effects of occupational exposure to organic solvents and noise on hearing. Scand. J. Work Environ. Health 19, 245-254.

Ohinataab, Y., Yamasoba, T., Schacht, J., Millers, J.M., 2000. Glutathione limits noise-induced hearing loss. Hear. Res. 146, 28-34.

Probst, R., Lonsbury-Martin, B.L., Martin, G.K., 1991. A review of otoacoustic emissions. J. Acoust. Soc. Am. 89, 2027-2067.

Puel, J.L., Bobbin, R.P., Fallon, M., 1988. An ipsilateral cochlear efferent loop protects the cochlea during intense sound exposure. Hear. Res. 37, 65-69.

Quirk, W.S., Shivapuja, B.G., Schwimmer, C.L., Seidman, M.D., 1994. Lipid peroxidation inhibitor attenuates noise-induced temporary threshold shifts. Hear. Res. 74, 217-220.

Ruan, R.S., Leong, S.K., Mark, I., Yeoh, K.H., 1999. Effects of BDNF and NT-3 on hair cell survival in guinea pig cochlea damaged by kanamycin treatment. Neuroreport 10, 2067-2071.

Schacht, J., 1993. Biochemical basis of aminoglycoside ototoxicity. Otolaryngol. Clin. N. Am. 26, 845-856.

Schmiedt, R.A., 1986. Acoustic distortion in ear canal. I. Cubic difference tones: effects of acute noise injury. J. Acoust. Soc. Am. 79, 1481-1490.

Segal, J.A., Harris, B.D., Kustova, Y., Basile, A., Skolnick, P., 1999. Aminoglycoside neurotoxicity involves NMDA receptor activation. Brain Res. 815, 270-277.

Segal, J.A., Skolnick, P., 1998. Polyamine-like actions of aminoglycosides and aminoglycoside derivatives at NMDA receptors. Eur. J. Pharmacol. 347, 311-317.

Sha, S.H., Schacht, J., 1999. Stimulation of free radical formation by aminoglycoside antibiotics. Hear. Res. 128, 112-118.

Sha, S.H., Schacht, J., 2000. Antioxidants attenuate gentamicin-induced free radical formation in vitro and ototoxicity in vivo: D-methionine is a potential protectant. Hear. Res. 142, 34- 40.

Shi, Y., Martin, W.H., 1997. ABR and DPOAE detection of cochlear damage by gentamicin. J. Basic Clin. Physiol. Pharmacol. 8, 141155.

Shoji, F., Miller, A.L., Mitchell, A., Yamasoba, T., Altschuler, R.A., Miller, J.M., 2000a. Differential protective effects of neurotrophins in the attenuation of noise-induced hair cell loss. Hear. Res. 146, 134-142.

Shoji, F., Yamasoba, T., Magal, E., Dolan, D.F., Altschuler, R.A., Miller, J.M., 2000b. Glial cell line-derived neurotrophic factor has a dose dependent influence on noise-induced hearing loss in the guinea pig cochlea. Hear. Res. 142, 41-55.

Smith, D.W., Erre, J.-P., Aran, J.-M., 1994. Rapid, reversible elimination of medial olivocochlear efferent function following single injections of gentamicin in the guinea pig. Brain Res. 652, 243248.

Staecker, H., Kopke, R., Malgrange, B., Lefebvre, P., Van de Water, T.R., 1996. NT-3 and/or BDNF therapy prevents loss of auditory neurons following loss of hair cells. Neuroreport 7, 889-894.

Sutton, L.A., Lonsbury-Martin, B.L., Martin, G.K., Whitehead, M.L., 1994. Sensitivity of distortion-product otoacoustic emissions in humans to tonal over-exposure: time course of recovery and effects of lowering $\mathrm{L}_{2}$. Hear. Res. 75, 161-174.

Tan, C.T., Lee, S.Y., Yao, C.J., Liu, S.H., Lin-Shiau, S.Y., 2001. Effects of gentamicin and $\mathrm{pH}$ on $\left[\mathrm{Ca}^{2+}\right]_{\mathrm{i}}$ in apical and basal outer hair cells from guinea pigs. Hear. Res. 154, 81-87.

Wang, Z., Li, H., 2000. Microglia-like cells in rat organ of Corti following aminoglycoside ototoxicity. Neuroreport 11, 1389-1393.

Yagi, M., Magal, E., Sheng, Z., Ang, K.A., Raphael, Y., 1999. Hair cell protection from aminoglycoside ototoxicity by adenovirus- 
mediated overexpression of glial cell line-derived neurotrophic factor. Hum. Gene Ther. 10, 813-823.

Yamane, H., Nakai, Y., Takayama, M., Konishi, K., Iguchi, H., Nakagawa, T., Shibata, S., Kato, A., Sunami, K., Kawakatsu, C., 1995. The emergence of free radicals after acoustic trauma and strial blood flow. Acta Otolaryngol. Stockh. 519 (Suppl.), $87-92$.
Yamasoba, T., Schacht, J., Shoji, F., Miller, J.M., 1999. Attenuation of cochlear damage from noise trauma by an iron chelator, a free radical scavenger and glial cell line-derived neurotrophic factor in vivo. Brain Res. 815, 317-325.

Young, J.S., Upchurch, M., Kaufman, M., Fechter, L.D., 1987. Carbon monoxide exposure potentiates high-frequency auditory threshold shifts induced by noise. Hear. Res. 26, 37-43. 